\title{
MAIMÓNIDES Y LEIBNIZ
}

\author{
Antonio Ríos Rojas \\ Universidad de Salamanca
}

\section{RESUMEN}

Es mi intención en este escrito mostrar los lugares comunes en la filosofía de un autor moderno como Leibniz y en la de un autor judío medieval como Maimónides. Trataré principalmente del concepto de substancia en ambos autores, viendo la similitud entre la mónada leibniziana y el concepto de «ángel» en Maimónides, ambos, «mónada» y «ángel», dirimen además del concepto de substancia, el de estructura de lo vivo, además de otras interesantes nociones cognoscitivas en las que trataremos de indagar.

Palabras clave: Leibniz, Maimónides, substancia.

\begin{abstract}
The aim of this writing is to show the similarities between the philosophy of a modern author, like Leibniz, and the philosophy of a medieval jewish author, like Maimonides. I am going to write about the concept of «substance» in both authors, looking into the similarity between the «monad» of Leibniz and the idea of «angel» of Maimonides. Both of them, «monad» and «angel», settle, not only the concept of «substance», but also the concept of «structure of living» besides some more interesting cognitive notions which I am going to inguire into.
\end{abstract}

Key words: Leibniz, Maimonides, Substance.

No es tan abismal la distancia que separa a la modernidad de la Edad Media, especialmente si nos fijamos en algunos modernos que de forma concisa no han querido romper radicalmente con el saber anterior, en cuanto éste implicaba una fuerte concepción metafísica del universo y del hombre como miembro de él. Un estudio pormenorizado de este particular sería interesante, pero a la vez muy extenso. De este forma, mi atención se centrará sólo en dos autores, uno medieval, Maimónides, y otro moderno, Leibniz. En concreto abordaré la relación de las mónadas en Leibniz con las concepciones maimonitas sobre el alma y la angelología.

En la historia de la filosofía no es difícil encontrar los lugares de disensiones y diferencias entre épocas y autores; más complejo, en cambio, es caer en la cuenta de los lugares comunes, y aún más complejo resulta ver la diferencia en lo común, quizás esto no se logre sin una idea de mundo, sin ese concepto cósmico de filosofía que exigiera Kant, o sin ese sentir heideggeriano de que, en el fondo, todos los pensadores piensan lo mismo, pero de distinta manera ${ }^{1}$.

Por estas razones sería muy fácil afirmar que el moralista Maimónides, el judío legalista, y el dualista radical, en sentido platónico fuerte, que fue el autor sefardí, se halla en absoluta 
discrepancia con el alemán de finales del XVII y principios del XVIII que fue Leibniz, quien esencialmente intenta fundir el desgajamiento y el dualismo cartesiano entre espíritu y materia en un concepto dialéctico, que anticipa los panteísmos decimonónicos y que es consecuencia de los semipanteísmos renacentistas.

Es cierto que, como suelen señalar la mayoría de los comentaristas leibnizianos, el autor alemán pretende otorgar al universo la unidad que le había negado Descartes. La división de la substancia en cogitans y extensa, otorgaba al hombre, en tanto que portador de la substancia pensante, un lugar central en el universo; al menos Descartes se erige en preparador de tal camino. La materia es sólo extensión, también movimiento, pero un movimiento meramente mecánico para Descartes. Desde esta perspectiva, la intención de Leibniz es devolver la unidad al universo, y devolver al hombre a un lugar más humilde dentro del macrocosmos. Este deseo le hace a Leibniz volver a considerar como válido el saber escolástico medieval, asintiendo con la validez del pensamiento en el mismo Tomás de Aquino ${ }^{2}$, validez que igualmente hubiera podido sostener respecto a Maimónides.

Esta reconsideración leibniziana de la filosofía implicaba una concepción viva y a la vez metafísica del mundo. En efecto, la metafísica ha sido siempre conexión viva, ya que pese a los fríos conceptos de forma, acto, potencia, substancia o accidente, latía en ella un concepto de la naturaleza que otorgaba a las cosas un principio interno, una esencia dinámica. Tales principios eran posibilitados bien gracias a un sistema de emanaciones continuas (neoplatonismo y gran parte del pensamiento árabe y judío), o bien por una línea más directamente creacionista. Pero, tanto por un lado, como por el otro, el mundo respiraba metafísicamente desde la idea de unidad, el mundo era un macrocosmos dentro del cual existían múltiples microcosmos. Esto dotaba a tales concepciones, por muy científicas que pretendieran mostrarse, de un carácter mágico y mistérico respecto al universo.

Quiero decir con esto, que en el mundo griego y en el mundo medieval, la unidad, la armonía, bien a nivel natural, bien a nivel de designación sobrenatural, era algo de lo que no cabía dudar. Esa unidad y armonía va perdiéndose con la división radical de órdenes y estamentos a partir del siglo XIV, y sólo hallará poso en el interior del hombre, él será el dador de la unidad a través de la conciencia, lo cual ocurre en la modernidad.

He aquí, pues, las dos líneas, una la medievalista, que lleva en su seno la tendencia a la unidad, otra la moderna, en la que la unidad sólo es posible a través de la conciencia pensante.

Leibniz se alza contra esto último. Su concepto de «mónada» unirá las dos substancias separadas con Descartes, y lo hará dotando a la realidad, a la naturaleza, de las facultades que antes habían sido asignadas al alma por Aristóteles. En el estagirita esto es muy claro, el alma vegetativa tiene principio propio y autónomo de movimiento, el movimiento no es meramente mecánico sino que se lo trata desde las fuerzas vivas que constituyen su esencia, el movimiento como soplo de vida ${ }^{3}$. Lo mismo ocurre con las almas sensitivas. Pero, no obstante, desvirtuaríamos al pensador griego haciéndole hablar del alma como meras fuerzas. Aristóteles nunca se muestra tan democritiano como algunos han pretendido verle:-Para él, el alma ya sea de una forma u otra, es una entidad, una forma del viviente. No hay átomos, está la forma y está la materia.

Por tanto, hay en Leibniz una recuperación de lo que, por un lado es Aristóteles, y, por otro, Demócrito. El mismo Leibniz, afirma que su sistema «combina Platón con Demócrito, Aristóteles con Descartes, los escolásticos con los modernos... los principios verdaderos de las

2 Cf. Leibniz, G: Discurso de Metafísica, Barcelona, Folio, 1999, af.11, p.76.

3 Esto es así en las almas vegetativas y sensitivas. Nótese que toda la crítica de Aristóteles al alma como armonía, como movimiento y como unión de contrarios (Cf. Aristóteles: De anima, cap. IV, Madrid, Gredos, 1994, p.152-157) se lleva a cabo tan sólo para criticar las concepciones del alma puramente humana, el entendimiento, el deseo y la voluntad. 
cosas los encuentro en las unidades de sustancia que dicho sistema introduce, y en su armonía mutua, preestablecida por la sustancia primitiva» ${ }^{4}$. La gran hazaña es otorgar a la realidad toda, a la naturaleza, una facultad que antes sólo había sido asignada al alma, es decir, la percepción, lo cual acontece mediante el concepto de mónada. Pero muy bien indica Leibniz que lo que se había asignado al alma era más bien la apercepción o conciencia ${ }^{5}$. «Y es en esto en lo que los cartesianos se han equivocado mucho, no habiendo tenido en cuenta para nada aquellas percepciones de las que uno no se da cuenta. Es esto también lo que les ha hecho creer que solamente los espíritus eran mónadas, y que no existían las almas de las Bestias ni otras entelequias» ${ }^{6}$.

Toda mónada tiene, por tanto, percepción, toda la realidad tiene, en tanto que constituida por mónadas, esta virtud que la extensión cartesiana le privó, la percepción. Pero efectivamente, las mónadas difieren entre sí en la cualidad, no en la cantidad, ya que son substancias simples? exentas de materia. De ahí que haya almas con apercepción o conciencia y otras con mera percepción. «Si queremos llamar Alma a todo lo que tiene percepciones y apetitos..., todas las substancias simples o mónadas creadas podrían ser también Almas; pero como el sentimiento es algo más que una simple percepción, concedo que el nombre general de mónadas y de Entelequias basta para las substancias simples que no tengan sino eso; y que se llaman Almas solamente a aquéllas cuya percepción es más distinta y está acompañada de memoria» ${ }^{8}$. Dar atributos de conciencia a lo que Descartes había quitado es el objetivo leibnizino.

Pero en este concepto de mónada anida también la lucha de Leibniz contra aquel medievalismo para el que sólo tiene percepción lo inteligente. Pasemos ya a considerar, desde esto último, a Maimónides, quien afirma que hay fuerzas corpóreas, pero que estas nada tienen que ver con la gran fuerza de lo no corpóreo, asentándose la fuerza de lo no corpóreo en la percepción de sus actos. Esas otras fuerzas corporales «constituyen una naturaleza, pero no tienen conciencia de su actividad»?.

El concepto leibniziano de alma es, en principio, portentosamente griego, tanto, que condensa como antes he apuntado, la cierta entidad de la que hablaba Aristóteles y el atomismo de Demócrito. Téngase en cuenta este equilibrio para ulteriores afirmaciones, ya que también se muestra en la lucha que dirimen la naturaleza lógica del ser en Maimónides y el atomismo creacionista de los pensadores mutacálimes.

Pero el concepto de mónada no se limita a designar una fuerza, el alma — la mónada a la que le compete la apercepción-, sino que también conoce, le compete, por tanto, la ciencia, la razón y los juicios. ${ }^{10}$ Tal como al alma aristotélica y tal como la concepción maimonídea del universo, para quien el hombre es un microcosmos inserto en el macrocosmos. Pero siendo un microcosmos que se da cuenta de los secretos de lo real. Si el hombre es microcosmos no lo es sólo por la perfección que muestra su organismo dentro de otro superior, no lo es sólo por la idea biológica de estructura, sino que lo es esencialmente por su facultad intelectiva. Pasaremos a verlo en Maimónides, pero véase la similitud de este pensamiento con estas palabras de Leibniz: «También es por el conocimiento de las verdades necesarias y por sus abstracciones por lo que somos elevados a los actos reflexivos, que nos hacen pensar en el llamado Yo, y considerar que esto o aquello se halla en nosotros; y ocurre que pensando en nosotros,

4 Leibniz, G: Nuevos ensayos sobre el entendimiento humano, Madrid, Editora Nacional, 1983, p. 70.

5 Cf. Leibniz, G: Monadología, ed citada, af. 14; p.25.

6 Ibid., p. 25-26.

7 Nótese, pese a todo, la diferencia con Demócrito, para quien las mónadas diferían en la cantidad, no en la cualidad.

8 Leibniz: o.c., af.19, p. 27. Cf. igualmente: Leibniz, G: Nuevos ensayos del entendimiento humano, ed. citada; p.149 y 154.

9 Maimónides: Guía de perplejos, Madrid, Trotta, 1994, II parte, cap.7, p. 256.

10 Cf. Leibniz: o.c., af. 29 , p. 30. 
pensamos en el Ser, en la Substancia, en lo simple y en lo compuesto, en lo inmaterial y en Dios mismo» ${ }^{11}$.

Como he indicado, en Maimónides, si el hombre es microcosmos, lo es por su entendimiento. El ser creado a imagen y semejanza de Dios no implica otra cosa sino que el alma es el punto de unión entre Dios y el hombre, y lo es en tanto que conoce, esto es, en tanto que entendimiento. El entendimiento reconoce sus límites y por conocerlos infiere que Dios, la Substancia primera de la cual proviene el entendimiento humano, carece de limitaciones. Así nos lo hace ver Maimónides en dos de los capítulos de la primera parte de su Guía de perplejos ${ }^{12}$. «Has de saber, lector de mi Tratado, que en las percepciones intelectuales, en cuanto dependientes de la materia, ocurre algo similar a lo inherente a las sensoriales. Así, mediante la visión ocular percibes algo asequible a esa facultad; pero si fuerzas tus ojos a hacer algo excesivo para tu capacidad, a mirar con fijeza o a gran distancia, más allá del límite de visión...violentando la visión con el fin de captar la auténtica realidad del objeto, tu vista se aminorará, no ya solamente para percibir lo que rebasa tu alcance, sino aquello mismo que te es accesible... sólo debe emplearse la inteligencia en lo asequible al hombre, pues tocante a materias que rebasan la perceptibilidad humana, es arriesgado abordarlas».

Así lo manifiesta también Leibniz, «se debe juzgar también que esa Substancia Suprema...es incapaz de límites ${ }^{13}$.

De este modo descubrimos como común en Maimónides y en Leibniz la idea de la existencia de límites. Esta idea es marcadamente medieval, y el racionalismo moderno no se libera de ella. En realidad toda filosofía posterior no se libera de esta idea. La única solución será marcar la imposibilidad al entendimiento humano de traspasar los límites, así lo intenta constitutivamente Kant, pero en realidad, el problema sólo se termina considerando como absurdo hablar de límites, lo cual implicaría que en efecto no es problema que haya o que deje de haber algo tras los límites. De esto se encarga buena parte del pensamiento contemporáneo.

Para Leibniz, pues, al igual que para Maimónides, se dan límites esenciales al entendimiento humano. Esto es lo que supone la comprensión sin límites de una Razón Suprema, y lo que supone en Leibniz un mundo de posibles. Leibniz no cierra, como tampoco Kant, la posibilidad en la física del recurso al infinito, a lo ilimitado ${ }^{14}$. Si Leibniz llega a afirmar que el espacio sin cuerpos no es nada sino la posibilidad de colocarlos, lo afirma precisamente porque es mediante el concepto de posibilidad, por el que el mundo no se cierra, no se limita. ${ }^{15}$. Hay, en efecto, un intento moderno de otorgar a la razón, en tanto que alma del hombre, unos principios estables, que cierren el mundo lógica y formalmente, por ello recurre Leibniz a un principio de razón suficiente, al cual se le confiere la tarea, en principio inmanente, de ser el eje a través del cual «consideremos que no podría hallarse ningún hecho verdadero o existente, ni ninguna enunciación verdadera, sin que haya una razón suficiente para que sea así y no de otro modo» ${ }^{16}$. Pero por si esto fuera poco, el autor alemán ha sentenciado poco antes que la mayoría de las razones suficientes no pueden ser conocidas por nosotros. ${ }^{17}$

1 Leibniz: o.c.,af.30, p.30.

12 Cf. Maimónides: o.c., I parte, cap.31-32, p.104.

13 Leibniz: o.c., af. 40 , p. 33.

14 Cf. Ibíd., af.36, p.32.

15 Este particular del problema de los límites en Leibniz está muy bien tratado en: Bennet, J: la dialéctica de la razón pura de Kant, Madrid, Alianza, 1981, esp. p.171-175. Pero podemos traer un texto del mismo Leibniz: «...la consideración de lo infinito e infinito se da siempre que haya magnitud y multiplicidad. El auténtico infinito no es una modificación, es lo Absoluto; por el contrario, siempre que se introduce una modificación, se limita, se forma un finito». (Leibniz, G: Nuevos ensayos sobre el entendimiento humano, ed. citada, p.182.

16 Leibniz: Monadología, ed. citada, af.32, p.31.

17 Cf. Ibíd., p.31. 
En el Discurso de Metafísica se muestra Leibniz abriendo caminos absolutamente nuevos, al sostener que, incluso los juicios, en tanto que son nuestros, nos engañan, tan sólo la percepción pura de las mónadas carecen de error ${ }^{18}$ en tanto que son creaciones y manifestaciones directas de Dios. Un nuevo camino, en efecto, se abre aquí, camino que será completado en la ontología por Hegel, en la lógica del corazón y de la percepción por Pascal, Rousseau y Kant. El hombre como centro gracias a su entendimiento, el hombre como microcosmos exclusivamente por su entendimiento va, en el proceso de la filosofía leibniziana dejando paso a una mayor consideración de lo orgánico, de lo vivo.

Pero debemos insistir en que pronto este principio de pretensiones inmanentes y orgánicas se difumina o se amplia — según se mire- en aspectos trascendentes al ser la Substancia Suprema (Dios) la garantía y el condicionante último del principio de razón suficiente. El argumento leibniziano es plenamente escolástico ya que sostiene que no es posible referir un principio claro de un hecho o de un acto presente, ya que implicaría un análisis infinito de detalles, por eso «es necesario que la razón suficiente o última esté fuera de la sucesión o series de este detalle de las contingencias por infinito que pueda ser»19.

Leibniz deja muy claro el camino de lo posible real (en Dios). Dios es el creador no sólo de la existencia, sino también de la esencia. Por ello las mónadas no pueden influir de forma determinante unas en otras, no hay aún una autonomía total de influencias en el universo. Las mónadas sólo influyen idealmente unas en otras ${ }^{20}$. Es el voluntarismo divino el que otorga o no esa unión. En este último caso, Leibniz se muestra como un atomista mutacálime, pero en el primer caso otorga fe a la posición maimonita, manteniendo así un equilibrio entre las dos posturas: por vía de Maimónides sostiene una naturaleza de lo posible ${ }^{21}$, otorgando, por el lado mutacálime, la continua efectividad por parte de la voluntad de Dios de lo posible. Eso sí, procura erradicar Leibniz lo que según Maimónides es el fundamental error mutacálime, la creación por parte de Dios de lo imposible.

Hasta ahora hemos intentado mostrar cómo se daba en Leibniz la idea de unos límites del entendimiento - aspecto muy medieval—, y cómo, por otro lado, defendía el autor alemán, la idea de infinito en lo físico. Hemos reparado también en la mónada fundamental que es el alma humana, y, ampliando nuestro recorrido, nos hemos fijado, a través de la concepción de la mera percepción, en la conexión necesaria entre las cosas, no necesariamente humanas, sino simplemente orgánica, mostrándose esto último como la verdadera esencia en estructura del mundo. A partir de estas ideas, vamos a aterrizar en la gran similitud del pensamiento maimonita, en lo que se refiere a la angelología, con las ideas leibnizianas.

Una máquina perfecta, eso es el mundo creado según Leibniz, y una máquina hasta en sus partes menores. Toda la materia es máquina y la máquina de la Naturaleza tiene hasta en sus partes ínfimas principio de movimiento ${ }^{22}$, tiene fuerza. La idea aristotélica del alma como principio del movimiento, idea que, como hemos mencionado, es rebajada por el estagirita por la idea de substancia, toma aquí cuerpo nuevamente. Esto le hace decir a Leibniz que cada porción de materia expresa el uñiverso, las mónadas son así espejos donde se refleja todo el mundo en ellas. Microcosmos y macrocosmos, esta es la idea expresada en esta bella imagen: «cada porción de la materia puede ser concebida como un jardín lleno de plantas, y como un estanque lleno de peces. Pero cada ramo de la planta, cada miembro del animal, cada gota de sus

18 Cf. Leibniz: Discurso de Metafisica, ed. citada, af.14, p. 81.

19 Ibid., p.32. Nótese cómo por ejemplo Santo Tomás rechaza sin más la idea de infinito en la primera vía, el infinito es imposible. No ocurre así en Leibniz,»...por infinito que pueda ser». Se nos vuelve a presentar Leibniz desde el equilibrio que mantiene su filosofía, es decir, aparece el infinito espacial, pero éste es incognoscible para el hombre finito.

20 Cf. Leibniz: o.c., af.51, p. 37.

21 Puede esto confrontarse en: Maimónides, o.c., I parte, cap.73, p. 218-220.

22 Cf. Leibniz: o.c., af.64-65, p. 42. 
humores, es a su vez, un jardín o un estanque semejante ${ }^{23}$. El concepto de estructura, de dialéctica estructural es el que se pone aquí en juego. Quizás sólo mentes con esta ansia holista y metafísica pueden realizar tales filosofías, sólo hombres analistas, pero no especialistas pueden dar al mundo este sentido.

La idea de estructura mantiene la diferencia entre las substancias o mónadas, no se trata de una estructura similar, ni siquiera de partes similares de la estructura. Cada mónada es distinta a otra, como hemos visto, en cualidad.

Hace notar Leibniz que de esta forma concibe Tomás de Aquino a los ángeles ${ }^{24}$. Pero Leibniz amplia esta diferencia en las substancias en base a su cualidad respecto a todo lo real, siendo cada substancia un espejo de Dios, y matiza Leibniz, «o bien, de todo el universo que cada una de ellas expresa a su manera». Esto nos hace considerar que la naturaleza, cada parte creada es reflejo de Dios. En la angelología de Aquino o del Maimónides ortodoxo, sólo los ángeles y las inteligencias contemplan y apetecen constantemente a Dios. En Leibniz, es toda la realidad la que se representa a su modo a Dios mismo, o a la estructura del universo. Pero la representación es diversa en cada mónada.

En Maimónides, los ángeles que, en un momento son identificados con las Inteligencias de las esferas, se representan y apetecen a Dios, pero el apetecer viene claramente de fuera, de Dios mismo, en cuanto objeto apetecido. En las mónadas no ocurre este venir de fuera, sólo en tanto que son creaciones de Dios tienen algo de fuera, pero son plenamente autónomas en su apetencia y en su representación, este es el sentido de la afirmación de que las mónadas no tienen ventanas.

Moses Ben Maimon, Maimónides trata la angelología de forma especial en su Guía de perplejos. Los apartados dedicados a la angelología en la Guía de perplejos son: I parte, cap.49; II parte, cap. 4-11; III parte, cap.1-7. En los capítulos de la segunda parte se comparan los ángeles con el intelecto activo y se intenta mostrar cómo los ángeles son fuerzas de movimiento. Pero es el capítulo sexto de esta segunda parte el que constituye una novedad respecto a la propia ortodoxia maimonita, y el que nos muestra ideas plenamente modernas respecto a la estructura metafísica del mundo. Lo iremos viendo. Los capítulos de la tercera III parte tratan de mostrar la correspondencia de la Escritura sobre los ángeles y la filosofía, especialmente la aristotélica.

Ya el análisis de la angelología ortodoxa maimonita refleja ideas posteriormente ampliadas por Leibniz y Spinoza, vamos a ver en primer término algunas características de esto que llamo angelología ortodoxa.

Los ángeles son definidos por el pensador cordobés como fuerzas ejecutoras del movimiento ${ }^{25}$. Nada se nos dice de que a través de ellos se refleje el mundo. Esto es así porque la autoconciencia -incluso la percepción- aún no es entendida como parte constitutiva y esencial del mundo. Con esto quiero insistir en que no se refleja el mundo a través del ángel, aunque esto no implique carencia de conciencia en el ángel. Existe conciencia, pero en el sentido plenamente medieval de inteligencia contemplativa de la realidad, y especialmente de la realidad de Dios, que como hemos indicado antes viene de fuera en forma de apetencia. Así, esa «fuerza ejecutora de movimiento» es acción, en base, no a lo que pudiera entenderse como fuerza orgánica, sino acción que supone la contemplación de Dios por parte de los ángeles.

23 Leibniz: Ibíd., af. 67, p.43.

24 Cf. Leibniz: Discurso de Metafísica, ed citada, af.9, p.74. Considérese que la angelología tomista, así como el tratamiento en el aquinate de la profecía, se halla fuertemente influenciado por las posiciones sobre ambos particulares de Maimónides, llegando de esta forma a Leibniz. Aunque se debe tener en cuenta que esta influencia también viene por vía de Spinoza. Para la influencia de la angelología maimonita en la de Aquino, Cf. Robles, L: Tomás de Aquino, lector de Maimónides; en: Maimónides. Actas del I Congreso intemacional, Córdoba, 1986, p.445-453.

25 Insisto en que voy a dirimir tan sólo los motivos metafísicos y ontológicos de la angelología, los motivos estrictamente gnoseológicos los he apuntado en: Ríos Rojas, A., Sobre angelología y profecia en Maimónides, en Revista española de filosofia medieval, 7, Zaragoza, 2000, p.141-152. 
Maimónides deja muy claro en primera instancia el carácter inmaterial de los ángeles, estos son inteligencias puras, para lo cual desmaterializa la figura del ángel ${ }^{26}$, ya que éste es un mediador entre el hombre y Dios, viniendo a ser identificado con el intelecto agente, que precisamente hace en gran parte del pensamiento medieval esta función mediadora. Pero también es un mediador entre la materia de las esferas y Dios, ya que el ángel es comparado con las Inteligencias separadas. Veamos algunos ejemplos de este primer tratamiento del ángel como mediador, contemplador e inteligencia pura. «Los ángeles tampoco están dotados de cuerpo, sino que son inteligencias separadas de la materia, pero que han sido hechos, Dios los creó».

Ya que el número de Inteligencias separadas es igual al número de esferas, Maimónides sostiene, apoyándose en Aristóteles, que tales inteligencias son ángeles, gracias a ellos las esferas se mueven, y lo hacen hacia Dios, dándose, además de ese movimiento de apetencia, representación, pero insisto, no representación del mundo a través de ella, como sería en Leibniz, sino representación de Dios y de los primeros principios:

«Las esferas son cuerpos vivos, dotados de alma e intelecto, que se representan y aprenden la divinidad, asi como también sus primeros principios; que existen las Inteligencias separadas absolutamente incorpóreas, todas emanadas de Dios, e intermediarias entre él y todos esos cuerpos celestes» ${ }^{27}$.

El ángel es ante todo —-desde este primer acercamiento de Maimónides- inteligencia pura, por ello se encuentra más cerca de Dios que de las criaturas mundanas. Este sentido jerárquico en los seres, que Maimónides debe sobre todo a Platón y también a la literatura bíblica respecto a los ángeles ${ }^{28}$, hará que lo material se considere una barrera, un velo para el intelecto, de este modo el conocimiento del hombre no puede ser perfecto, por su inmersión en ella. El ángel, en cambio, por estar exento de materia puede conocer, contemplar, siendo esa su esencia. Hasta este momento, toda comparación del ángel con la mónada leibniziana sería amplitud imaginativa. Pero sigamos más adelante.

Respecto al movimiento de los ángeles, afirma Maimónides que la dificultad que hubiera Ilevado a compararlos con Dios ha hecho que se les imagine bajo figura humana y con movimiento ${ }^{29}$, bien que para expresar el movimiento perfecto, se le asigna el único atributo perfecto en los seres irracionales, que es además el movimiento más noble en ellos, el vuelo y las alas, para así expresar mejor su equiparación a la esencia divina, y su mayor proximidad a Dios que al hombre ${ }^{30}$. Pero en realidad el movimiento del ángel es de extrema importancia en el orden metafísico para el autor sefardí, «mediante su mediación se movilizan las esferas —causa de la generación de lo que nace-... nunca encontrarás realice Dios acto alguno sino por ministerio de un ángel ${ }^{31}$. Maimónides se muestra aquí inserto en la tradición aristotélica y árabe, en la que las esferas tienen movimiento por tener almas, siendo la fuerza del ángel en cuanto inteligente el que mueve directamente a las esferas.

Hasta ahora tenemos dos ganancias fundamentales, una la no materialidad de los ángeles, otra, la de ser intermediarios y poner en acto a las esferas. Todo esto indica que, aunque existe un sistema de jerarquía y de emanación en el universo, la respectividad de todos los elementos del mismo no es total. Dicho de otro modo, el «sistema» no es aún dueño y controlador del universo. Claro que hay cierta armonía, pero las jerarquías mandan. La división clara ller, 1977

29 Cf. Maimónides: o.c., I parte, cap.49, p.137.

30 Cf. Ibíd., II parte, cap.6, p.254

30 Cf. Ibíd., II parte, cap.6, p.254

Cf. Maimónides: o.c., I parte, cap.49.

Ibíd., II parte, cap.4, p.251.

Cf. a este respecto: Gonzalo Rubio, C: La angelología en la literatura rabínica y sefardí. Barcelona, Ame- 
que sostiene que el espíritu es diferente, más perfecto y más divino que la materia lo deja bien claro. En cambio, en Leibniz el sistema comienza a tener una importancia decisiva y explícita, por ello los conceptos de materia y espíritu pueden andar en una armonía mayor que en el mundo medieval, armonía que otorga un entendimiento perfecto entre materia y espíritu, entendimiento que puede ocasionar la difuminación de ambos conceptos a nivel ontológico. «Este sistema hace que los cuerpos áctúen como si no hubiera almas; y que las almas actúen como si no hubiera cuerpos; y que ambos actúen como si el uno influyera sobre el otro»32.

Esto es posible por el mayor dinamismo y movimiento que da Leibniz a su sistema, dinamismo y movimiento que provienen de lo que llama Fulguración ${ }^{33}$ y que no es otra cosa que emanación continua, de hecho así lo denomina el autor en otro lugar ${ }^{34}$, Dios crea continuamente, ese crear se encuentra en la armonía preestablecida. Parecería que el continuo crear anula toda necesariedad o naturaleza del ser, pero no es así ya que la continua creación forma parte del plan divino en Leibniz. En cambio, la emanación medieval es mucho más estática, como he dicho antes, jerárquica. Claro que esto es así porque el principal fin de Maimónides y del medievalismo es la contemplación, y es desde este radical concepto desde el que los ángeles y toda esencia mediadores interviene en el proceso. La modernidad ya no tendrá como fin máximo este ver a Dios, sino manifestar, sacar los procesos vivos de la naturaleza, bien para darles un sentido divino o mundano.

Pero no olvidemos que al principio de este escrito sosteníamos que era tarea menos ardua comparar en base a diferencias que hacerlo en base a similitudes. Pero esto es así porque las similitudes, aunque existentes son escasas. La dificultad no estriba en excesivas comparaciones y relaciones sino en que hay autores que sólo brevemente apuntan caminos nuevos. Maimónides se nos muestra abriendo este camino cuando afirma en el anteriormente señalado capítulo 6 de la I parte de la Guía de perplejos, donde dice:: «...incluso los menores elementos del universo, hasta la formación de los miembros del animal, tal como son han sido hechos por intermediarios de los ángeles, puesto que todas las facultades son ángeles... i Cuánta es la ceguera de la ignorancia y cuán peligrosa! Si dijeras a uno de esos sedicentes sabios de Israel que Dios envía su ángel para que forme en el seno de la mujer un feto, le parecería maravillosamente, y lo aceptaría, considerándolo como una manifestación del poder divino, así como de su sabiduría...Pero si le dices que Dios puso en el semen una fuerza formativa que modela y estructura esos miembros y eso es el ángel, o bien que todas las estructuras proceden de la acción del intelecto activo, y ése es el ángel y el príncipe del mundo de quien tanto hablan los Doctores, lo rechazaria, como incapaz de comprender el sentido de esa auténtica grandeza y poderío consistentes en la efectuación de fuerzas activas en una cosa, imperceptibles al sentido. Los Doctores ( $j$ bendita sea su memoria!) Ya expusieron, para el verdadero sabio, que cada una de las fuerzas corporales es un ángel, cuanto más las que están difundidas por el universo, y que cada una de ellas posee determinada actividad, no dos ${ }^{35}$.

Nótese la gran idea inserta en el texto que nos lleva incluso más allá de Leibniz, en ningún momento dice Maimónides que esa fuerza que es el ángel es ahora consciencia o percepción. Se trata de mera fuerza ejecutora de movimiento en vistas a alguna función determinada. Esto es radicalmente distinto a aquella consideración del ángel como fuerza ejecutora de movimiento de las esferas, en base a la comprensión y a la conciencia. Es sin duda el médico Maimónides quien puede decir esto. Por ello estas páginas son las más sorprendentes del autor judío. Maimónides habla de fuerza para enterrar su propio concepto de ángel espiritual que tiene como fin la contemplación. Fuerza que según manifiesta el texto es de portentoso carác-

Leibniz: o.c., af.81, p.47.

Cf. Ibíd., af.47, p.36.

Cf. Leibniz: Discurso de Metafisica, ed. citada af.14, p.81.

35 Maimónides: o.c., Il parte, cap.6, p.255. 
ter biológico. Habla también de «fuerzas» para dar nueva luz a aquello que había sostenido respecto a la percepción de las esferas y a la división del mundo superior e inferior, siendo este último algo muerto en tanto que portador de los cuatro elementos y siendo algo vivo el mundo superior, por estar dirigido por las inteligencias de las esferas. ${ }^{36}$

Sigue avanzando Maimónides al afirmar que esas fuerzas realizan una actividad determinada, no dos, lo cual hace caer en la cuenta del puro estructuralismo de lo vivo, en este caso, más acentuado que en el mismo Leibniz, donde la mónada, más que función tiene conciencia, o mejor dicho, percepción. «En el Beresit R. leemos: Se ha enseñado que un sólo ángel no efectúa dos misiones, ni dos realizan una misma. Tal es el carácter de todas las fuerzas. Esto te confirmará en que todas las energías individuales, tanto fisicas como psíquicas, se denominan ángeles»37.

Si Maimónides emplea este concepto de fuerza aplicado al ángel, también Leibniz lo emplea para combatir el mecanicismo cartesiano, para quien los cuerpos tenían extensión y movimiento mecánico. La incursión de la fuerza es con fines no sólo de explicación física, sino también metafísica. «Esta fuerza es algo diferente de la magnitud, de la figura y del movimiento y de ello puede concluirse que no todo lo que se concibe en el cuerpo consiste únicamente en la extensión y en sus modificaciones, como creen nuestros modernos. Así, nos vemos forzados a restablecer algunos seres oformas que ellos han desterrado. Y cada vez más parece que, aunque todos los fenómenos particulares de la Naturaleza se puedan explicar matemática o mecánicamente por los que los entienden, los principios generales de la naturaleza corporal y de la mecánica misma son más bien metafísicos que geométricos y corresponden más bien a algunas formas o naturalezas indivisibles, como causas de las apariencias, que a la masa corporal o extensión ${ }^{38}$.

En último lugar debemos considerar el hecho de si los ángeles son o no perecederos. Contra la opinión común de que efectivamente los ángeles son vivientes permanentes, Maimónides sostiene que algunos son perecederos y otros no. Cabría pensar, según esto, que pudiera hacer ahora matices cualitativos sobre las realidades angélicas, desde los cuales inserte diferencias entre el semen antes citado y el ángel en cuanto intermediario o el ángel en cuanto entendimiento. Pero el autor sefardí nos sorprende al afirmar, que, en efecto, hay perecederos y hay no perecederos, «y tal es la realidad, puesto que las fuerzas individuales nacen y mueren de continuo, en tanto que las especies correspondientes son perdurables, sin deterioro ${ }^{39}$.

Esto nos indica que sólo la especie, que también es angélica, en tanto que sus partes lo $\operatorname{son}^{40}$, se muestra en condiciones reales de no perecer. El sistema es lo que da sentido, pues, a las partes. Este es un sentido incluso más averroísta que leibniziano. Sólo la especie es inmortal, intelecto agente para todos y separado. Con esto es difícil no ver en estas páginas del pensador judío un materialismo preñado de idealismo radical al modo de Spinoza.

Antonio Ríos Rojas

Apdo. de correos 255

02630 La Roda (Albacete)

\footnotetext{
36 Cf. Ibíd., II parte, cap.5, p.252.

37 Ibíd., II parte, cap.6, p.255.

38 Leibniz: Discurso de Metafisica, p.88.

39 Maimónides: 0.c, p.255.

40 Nótese la similitud con Leibniz (Cf. Leibniz: Discurso de metafísica, ed. citada p 89-91.; Monadología, ed. citada, p. 25 y 36 especialmente.
} 